J. Korean Math. Soc. 49 (2012), No. 5, pp. 1031-1051

http://dx.doi.org/10.4134/JKMS.2012.49.5.1031

\title{
THE CONNECTED SUBGRAPH OF THE TORSION GRAPH OF A MODULE
}

\author{
Shaban Ghalandarzadeh, Parastoo Malakooti Rad, and Sara Shirinkam
}

\begin{abstract}
In this paper, we will investigate the concept of the torsiongraph of an $R$-module $M$, in which the set $T(M)^{*}$ makes up the vertices of the corresponding torsion graph, $\Gamma(M)$, with any two distinct vertices forming an edge if $[x: M][y: M] M=0$. We prove that, if $\Gamma(M)$ contains a cycle, then $\operatorname{gr}(\Gamma(M)) \leq 4$ and $\Gamma(M)$ has a connected induced subgraph $\bar{\Gamma}(M)$ with vertex set $\left\{m \in T(M)^{*} \mid \operatorname{Ann}(m) M \neq 0\right\}$ and $\operatorname{diam}(\bar{\Gamma}(M)) \leq$ 3. Moreover, if $M$ is a multiplication $R$-module, then $\bar{\Gamma}(M)$ is a maximal connected subgraph of $\Gamma(M)$. Also $\bar{\Gamma}(M)$ and $\bar{\Gamma}\left(S^{-1} M\right)$ are isomorphic graphs, where $S=R \backslash Z(M)$. Furthermore, we show that, if $\bar{\Gamma}(M)$ is uniquely complemented, then $S^{-1} M$ is a von Neumann regular module or $\bar{\Gamma}(M)$ is a star graph.
\end{abstract}

\section{Introduction}

In [11], Beck introduced and investigated the zero-divisor graph of a commutative ring. He let all elements of the ring be vertices of the graph. In [8], Anderson and Livingston introduced and studied a zero-divisor graph, whose vertices are non-zero zero-divisors while, $x-y$ is an edge whenever $x y=0$. Since then, the concept of zero-divisor graphs has been studied extensively by many authors; see $[2,6,7,10]$. The concept of a zero-divisor graph has been extended to non-commutative rings by Redmond [21]. This concept also has been introduced and studied for semigroups by DeMeyer, McKenzie and Schneider in [13], and for near-rings by Cannon et al, in [12]. For recent developments on graphs of commutative rings see $[3,4,5,17]$.

Let $R$ be a commutative ring with identity element and $M$ be a unitary $R$ module. In this research, we will investigate the concept of the torsion-graph of an $R$-module $M$, which has been defined in [16]. The torsion graph $\Gamma(M)$ of $M$ is a simple graph, whose vertices are the non-zero torsion elements of $M$, and two distinct elements $x, y$ are adjacent if and only if $[x: M][y: M] M=0$. The residual of $R x$ by $M$, is denoted by [ $x: M]$, is the set of elements $r \in R$

Received April 12, 2011; Revised January 13, 2012.

2010 Mathematics Subject Classification. 13A99, 05C99, 13 C99.

Key words and phrases. torsion graph, multiplication modules, von Neumann regular modules. 
such that $r M \subseteq R x$ for $x \in M$. The annihilator of an $R$-module $M$, denoted by $\operatorname{Ann}_{R}(M)$, is $[0: M]$.

An $R$-module $M$ is called a multiplication module if for every submodule $K$ of $M$ there exists an ideal $I$ of $R$ such that $K=I M$ (Barnard [10]). A proper submodule $N$ of $M$ is called a prime submodule of $M$, whenever $r m \in N$ (where $r \in R$ and $m \in M$ ) implies that $m \in N$ or $r \in[N: M]$.

An $R$-module $M$ is called a cancellation module if $I M=J M$ for any ideals $I$ and $J$ of $R$ implies that $I=J$. Also, an $R$-module $M$ is a weak-cancellation module if $I M=J M$ for any ideals $I$ and $J$ of $R$ implies that $I+\operatorname{Ann}(M)=$ $J+\operatorname{Ann}(M)$. Finitely generated multiplication modules are weak cancellation, Theorem 3 [1].

Let $T(M)$ be the set of elements of $M$ such that $\operatorname{Ann}(m) \neq 0$. It is clear that if $R$ is an integral domain, then $T(M)$ is a submodule of $M$, which is called the torsion submodule of $M$. If $T(M)=0$, then the module $M$ is said to be torsion-free, and it is called the torsion module if $T(M)=M$. Thus $\Gamma(M)$ is an empty graph if and only if $M$ is a torsion-free $R$-module. We use the symbol $\bar{\Gamma}(M)$ to show the induced subgraph $\Gamma(M)$ with vertex set $\left\{m \in T(M)^{*} \mid \operatorname{Ann}(m) M \neq 0\right\}$. In this paper, we will also investigate the interplay of module properties of $M$ in relation to the properties of $\bar{\Gamma}(M)$. We believe that this study helps to illuminate the structure of $T(M)$. For example, if $M$ is a multiplication $R$-module, we show that $M$ is finite if and only if $\bar{\Gamma}(M)$ is finite. Recall that a graph is finite if both its vertices set and edges set are finite. We know that a graph $G$ is connected if there is a path between any two distinct vertices. The distance $d(x, y)$ between connected vertices $x, y$ is the length of the shortest path from $x$ to $y(d(x, y)=\infty$ if there is no such path). The diameter of $G$ is the diameter of a connected graph, which is the supremum of the distances between vertices. The diameter is 0 if the graph consists of a single vertex. The girth of $G$, denoted by $\operatorname{gr}(G)$, is defined as the length of the shortest cycle in $G(\operatorname{gr}(G)=\infty$ if $G$ contains no cycles).

A ring $R$ is called reduced if $\operatorname{Nil}(R)=0$. An $R$-module $M$ is called a reduced module if $r m=0$ for $r \in R$ and $m \in M$, implies that $r M \cap R m=0$. Also a $\operatorname{ring} R$ is von Neumann regular if for each $a \in R$ there exists an element $b \in R$ such that $a=a^{2} b$. It is clear that every von Neumann regular ring is reduced. Recall that a ring $R$ is called Bézout if every finitely generated ideal $I$ of $R$ is principal. We know that every von Neumann regular ring is Bézout.

A submodule $N$ of $M$ is called a pure submodule of $M$ if $I M \cap N=I N$ for every ideal $I$ of $R$ (Ribenboim in [22]). In [18], Kash (p. 105) states that an $R$-module $M$ is called a von Neumann regular module if and only if every cyclic submodule of $M$ is a direct summand in $M$. If $N$ is a direct summand in $M$, then $N$ is pure but not conversely (see [20], Example 2, p. 54 and [22], Example 14, p. 100). Therefore every von Neumann regular module is reduced.

A complete graph is a simple graph whose vertices are pairwise adjacent, and the complete graph with $n$ vertices is denoted by $K_{n}$. A bipartite graph is one whose vertex set can be partitioned into two subsets so that no edge has 
both ends in the same subset. A complete bipartite graph is one in which each vertex is joined to every vertex that is not in the same subset; the complete bipartite graph, with two parts of sizes $m$ and $n$, is denoted by $K_{m, n}$. The complete bipartite graph $K_{1, n}$ is called a star.

Let $G$ be a graph and $V(G)$ denote the vertices of $G$. Let $v \in V(G)$, as in [7]; $w \in V(G)$ is called a complement of $v$, if $v$ is adjacent to $w$ and no vertex is adjacent to both $v$ and $w$. That is, the edge $v-w$ is not an edge of any triangle in $G$. In this case, we write $v \perp w$. In module-theoretic terms, for multiplication $R$-module $M$, this is the same as saying $v \perp w$ in $\Gamma(M)$ if and only if $v, w \in T^{*}(M)$ and $\operatorname{Ann}(w) M \cap \operatorname{Ann}(v) M \subset\{0, v, w\}$. Moreover, we will follow the authors in [7] and say that $G$ is complemented if every vertex has a complement, and it is uniquely complemented if it is complemented and any two complements of the vertices set are adjacent to the same vertices. From Theorems 3.5 and 3.9 [7], we know that for a ring $R$ with non-zero nilpotent elements, $\Gamma(R)$ is uniquely complemented if and only if $\Gamma(R)$ is a star graph. If $R$ is reduced and $\Gamma(R)$ is complemented, then $S^{-1} R$ is a von Neumann regular ring, where $S=R \backslash Z(R)$.

In Section 2, we give an example of non-isomorphic modules with the same torsion graph. We show that $\bar{\Gamma}(M)$ is always connected with $\operatorname{diam}(\bar{\Gamma}(M)) \leq 3$. Furthermore, we prove that if $\Gamma(M)$ contains a cycle, then $\operatorname{gr}(\Gamma(M) \leq 4$. In this manner, we study some of the properties of $\bar{\Gamma}(M)$, when $M$ is a multiplication $R$-module. An $R$-module $M$ is a multiplication module if for every submodule $K$ of $M$ there exists an ideal $I$ of $R$ such that $K=I M$. It is clear that if $M$ is a multiplication $R$-module, then $\bar{\Gamma}(M)$ is a maximal connected subgraph of $\Gamma(M)$.

In Section 3, we obtain $\bar{\Gamma}(M) \cong \bar{\Gamma}\left(S^{-1} M\right)$, where $S=R \backslash Z(M)$ if $M$ is an $R$-module such that $\operatorname{Ann}(x)=\operatorname{Ann}([x: M] M)$ for all $x \in T(M)$.

In Section 4, we investigate complemented and uniquely complemented torsion graphs. We also extend Theorem 3.9 of [7] to the multiplication $R$-modules. Furthermore, for a multiplication $R$-module $M$ when $R$ is Bézout or cyclic $R$ module and prove that if $\bar{\Gamma}(M)$ is uniquely complemented, then either $\bar{\Gamma}(M)$ is a star graph or $S^{-1} M$ is a von Neumann regular module, where $S=R \backslash Z(M)$.

Throughout the paper, we use the symbol $(x, y)$ or $x+y$ to denote the elements of $M=M_{1} \oplus M_{2}$ and $T(M)^{*}=T(M) \backslash\{0\}$. Also, we use the symbol $(M)_{R}$ to denote $M$ as an $R$-module. Let $Z(M):=\{r \in R \mid r m=0$ for some $0 \neq m \in M\} . N i l(R)$ is an ideal consisting of nilpotent elements of $R$,

$$
N i l(M):=\cap_{N \in \operatorname{Spec}(M)} N
$$

$\operatorname{Spec}(M)$ is a set of the prime submodules of $M$, and for submodule $N$ of $M$, $D(N):=\left\{n \in N \mid[n: M]\left[n^{\prime}: M\right] M=0\right.$ for some non-zero $\left.n^{\prime} \in M\right\}$. As usual, the rings of integers and integers modulo $n$ will be denoted by $\mathbb{Z}$ and $\mathbb{Z}_{n}$, respectively. 


\section{Properties of $\bar{\Gamma}(M)$}

In this section, we show that $\bar{\Gamma}(M)$ is connected and has a small diameter and girth, and for a multiplication $R$-module $M$ with $|M| \geq 5$, we prove that if $\bar{\Gamma}(M)$ is complete, then $\operatorname{Nil}(M)=V(\Gamma(\bar{M})) \cup\{0\}$. We begin with the following example which shows that non-isomorphic modules may have the same torsion graphs.

Example 2.1. Let $M=M_{1} \oplus M_{2}$ be an $R$-module, where $M_{1}$ is a torsion-free module. So $T(M)^{*}=\left\{\left(0, m_{2}\right) \mid m_{2} \in T\left(M_{2}\right)^{*}\right\}$ and $\left[\left(0, m_{2}\right): M\right]=0$. Hence $\Gamma(M)$ is a complete graph. Let $M=\mathbb{Z} \oplus \mathbb{Z}_{n}$ be a $\mathbb{Z}$-module, so $\Gamma(M)=K_{n-1}$ for $n \geq 2$.

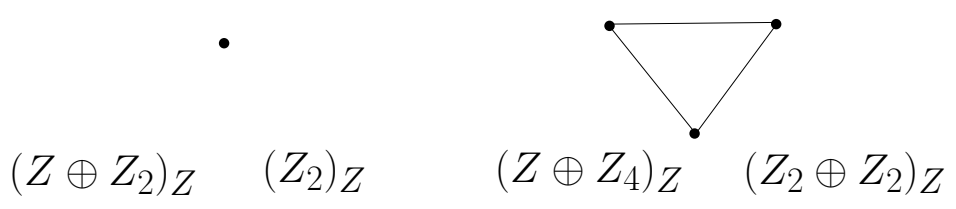

FiguRe 1

We know that $\Gamma(M)$ may be infinite (that is, the $R$-module $M$ has infinitely torsion elements). An interesting case occurs when $\Gamma(M)$ is finite, because in the finite case a drawing of the graph is possible. The next theorem shows that for a multiplication $R$ - module $M, \bar{\Gamma}(M)$ is finite (except when $\bar{\Gamma}(M)$ is empty) if and only if $M$ is finite.

Theorem 2.2. Let $M$ be a multiplication $R$-module. Then $\bar{\Gamma}(M)$ is finite if and only if either $M$ is finite or $V(\bar{\Gamma}(M))=\emptyset$.

Proof. Suppose that $\bar{\Gamma}(M)$ is finite and nonempty. Then there exists $x \in$ $V(\bar{\Gamma}(M))$; let $N=R x$ and $0 \neq s m \in R x$, where $s \in[x: M]$ and $m \in M$, so $0 \neq \operatorname{Ann}(x) M \subseteq \operatorname{Ann}(n) M$ for all $n \in N$. Hence $N \subseteq V(\bar{\Gamma}(M))$. Therefore $N$ is finite. Now if $M$ is infinite, then there is an element $n \in N$ such that $H=\{m \in M \mid s m=n\}$ is infinite. For all distinct elements $m_{1}, m_{2} \in H$, $s m \in \operatorname{Ann}\left(m_{1}-m_{2}\right) M$. So $m_{1}-m_{2} \in V(\bar{\Gamma}(M))$ is a contradiction. Thus $M$ is finite.

Corollary 2.3. Let $M$ be a multiplication $R$-module. Then $\Gamma(M)$ is finite if and only if either $M$ is finite or $M$ is a torsion-free $R$-module.

Proof. If $\Gamma(M)$ is finite, then $\bar{\Gamma}(M)$ is finite. Therefore by Theorem 2.2 either $M$ is finite or $V(\bar{\Gamma}(M))=0$. Thus for all $x \in M$, $\operatorname{Ann}(x) M=0$; hence $\operatorname{Ann}(x)=\operatorname{Ann}(M)$ for all $x \in M$. Now if $M$ is faithful, then $M$ is torsion-free; otherwise $T(M)=M$. Consequently, either $M$ is finite or $M$ is a torsion-free $R$-module. 

fluous.

The following example shows that the multiplication condition is not super-

Example 2.4. Let $R=\mathbb{Z}$ and $M=\mathbb{Z} \oplus \mathbb{Z}_{n}$. Clearly $M$ is faithful and is not finite, but by Example 2.1, $\bar{\Gamma}(M)=K_{n-1}$ is finite.

Now we show that for all $R$-module $M, \bar{\Gamma}(M)$ is connected with diameter $\leq 3$.

Theorem 2.5. Let $M$ be an $R$-module. Then

$$
\bar{\Gamma}(M) \text { is connected with } \operatorname{diam}(\bar{\Gamma}(M)) \leq 3 \text {. }
$$

Moreover, if $\bar{\Gamma}(M)$ contains a cycle, then $\operatorname{gr}(\bar{\Gamma}(M)) \leq 7$.

Proof. Let $x, y \in V(\bar{\Gamma}(M))$ be two distinct elements. If $[x: M] M$ or $[y$ : $M] M$ or $[x: M][y: M] M$ is zero, then $d(x, y)=1$. Therefore we suppose that $[x: M] M,[y: M] M$, and $[x: M][y: M] M$ is non-zero, so there are non-zero elements $\alpha \in[x: M][y: M]$ and $m \in M$ such that $\alpha m \neq 0$. If $[x: M]^{2}=[y: M]^{2}=0$, then $\alpha m \in V(\bar{\Gamma}(M))$, and hence $x-\alpha m-y$ is a path of length 2. Hence suppose that $[x: M]^{2}=0$ and $[y: M]^{2} \neq 0$; since $y \in V(\bar{\Gamma}(M))$, there exist non-zero elements $s \in \operatorname{Ann}(y)$ and $m_{0} \in M$ such that $s m_{0} \neq 0$. Now we consider the case $[x: M] \operatorname{Ann}(y) M=0$. In this case $s m_{0} \in V(\bar{\Gamma}(M))$, so $x-s m_{0}-y$ is a path of length 2. In the other case, if $[x: M] \operatorname{Ann}(y) M \neq 0$, then $m_{1}:=\alpha_{1} t m \in V(\bar{\Gamma}(M))$ for some nonzero elements $\alpha_{1} \in[x: M], t \in \operatorname{Ann}(y), m \in M$, and $x-m_{1}-y$ is a path of length 2. A similar argument holds if $[x: M]^{2} \neq 0,[y: M]^{2}=0$. Thus we may assume that $[x: M]^{2},[y: M]^{2}$ and $[x: M][y: M]$ are all non-zero. If $\operatorname{Ann}(x) \nsubseteq \operatorname{Ann}(y)$ and $\operatorname{Ann}(y) \nsubseteq \operatorname{Ann}(x)$, then there exist non-zero elements $r, s \in R$ such that $r x=0, r y \neq 0$ and $s x \neq 0, s y=0$, hence $r y, s x \in V(\bar{\Gamma}(M))$. Now if $r y \neq s x$, then $x-r y-s x-y$ is a path of length 3 . In the other case, if $r y=s x$, then $x-r y-y$ is a path of length 2 . Therefore $d(x, y) \leq 3$. Thus we may assume that $\operatorname{Ann}(x) \subseteq \operatorname{Ann}(y)$ or $\operatorname{Ann}(y) \subseteq \operatorname{Ann}(x)$. If $\operatorname{Ann}(x) \subseteq \operatorname{Ann}(y)$, then $r m \in V(\bar{\Gamma}(M))$ for some $r \in \operatorname{Ann}(x), m \in M$ and $x-r m-y$ is a path of length 2. A similar argument holds if $\operatorname{Ann}(y) \subseteq \operatorname{Ann}(x)$. Hence $d(x, y) \leq 3$; thus $\operatorname{diam}(\Gamma(\bar{M})) \leq 3$. If $\bar{\Gamma}(M)$ contains a cycle, by Proposition $1.3[14]$, then $\operatorname{gr}(\bar{\Gamma}(M)) \leq 7$.

As an immediate consequence, we obtain the following result.

Corollary 2.6. Let $M$ be a faithful $R$-module. Then $\Gamma(M)$ is connected with $\operatorname{diam}(\Gamma(M)) \leq 3$.

The following example shows that the faithful condition is not superfluous.

Example 2.7. Let $R=\mathbb{Z}$ and $M=\mathbb{Z}_{2} \oplus \mathbb{Z}_{3}$; then $\Gamma(M)$ is not connected.

Proposition $1.3[14]$ and Corollary 2.6 show that $\operatorname{gr}(\Gamma(M)) \leq 7$, when $\Gamma(M)$ contains a cycle. We next improve this sentence to $\operatorname{gr}(\Gamma(M)) \leq 4$. 




Figure 2

Theorem 2.8. Let $M$ be a multiplication $R$-module. If $\Gamma(M)$ contains a cycle, then $\operatorname{gr}(\Gamma(M) \leq 4$.

Proof. Let $m_{0}-m_{1}-m_{2}-\cdots-m_{n}-m_{0}$ be the shortest cycle of $\Gamma(M)$ for $n \geq 4$. If $\left[m_{1}: M\right]\left[m_{n-1}: M\right] M=0$, then $\Gamma(M)$ contains a cycle $m_{1}-m_{2}-\cdots-m_{n-1}$ $-m_{1}$, which is a contradiction. So there exist non-zero elements $\alpha \in\left[m_{1}: M\right]$, $\beta \in\left[m_{n-1}: M\right]$, and $m \in M$ such that $\alpha \beta m \in V(\Gamma(M))$. If $\alpha \beta m \neq m_{0}$ and $\alpha \beta m \neq m_{n}$, then $\Gamma(M)$ contains a cycle $m_{0}-\alpha \beta m-m_{n}-m_{0}$ is a contradiction. Therefore $\alpha \beta m=m_{0}$ or $\alpha \beta m=m_{n}$. So without loss of generality, assume $\alpha \beta m=m_{0}$; thus $\left[m_{0}: M\right] m_{0}=0$. Now we show that $R m_{0}=\left\{0, m_{0}\right\} \subset$ $R m_{1}$. If there exists a non-zero element $x \in R m_{0}$ such that $x \neq m_{0}$ and $x \neq m_{1}$, then $m_{0}-m_{1}-x-m_{0}$ is a cycle of length 3 , which is a contradiction. Hence $R m_{0}=\left\{0, m_{0}\right\} \subset R m_{1}$. Therefore there exists $y \in R m_{1}$ such that $y \neq 0$ and $y \neq m_{1}$. By a routine argument we obtain $y \neq m_{0}$ and $y \neq m_{2}$; therefore $m_{0}-m_{1}-m_{2}-y-m_{0}$ is a cycle of length 4 , which is a contradiction. Consequently, $\operatorname{gr}(\Gamma(M) \leq 4$.

Theorem 2.9. Let $M$ be a multiplication $R$-module. If $\bar{\Gamma}(M)$ is complete, then either $|M|=4$ or $\operatorname{Nil}(M)=V(\bar{\Gamma}(M)) \cup\{0\}$.

Proof. First suppose that $[x: M]^{2} M \neq 0$ for some $x \in V(\bar{\Gamma}(M))$, so $x \notin$ $\operatorname{Ann}(x) M$. In this case, we show that $|M|=4$. Put $N:=\operatorname{Ann}(x) M$. We divide the proof of the theorem into 6 claims, which are of some interest in their own right.

Claim $1: N$ is a prime submodule of $M$. Since $x \notin \operatorname{Ann}(x) M, N$ is a proper submodule of $M$. Let $r m \in N$ and $m \notin N$; here $r$ and $m$ denote elements of $R$ and $M$, respectively. Accordingly, $r[m: M][x: M] M=0$, so $r k x=0$ for all $k \in[m: M]$ and $r \in \operatorname{Ann}(k x)$. But there exists $k_{0} \in[m: M]$ such that $k_{0} x \in V(\bar{\Gamma}(M))$; consequently, $\operatorname{Ann}\left(k_{0} x\right) M \subseteq \operatorname{Ann}(x) M$. Thus $r M \subseteq N$ and $r \in[N: M]$. Therefore $N$ is a prime submodule, and as a consequence $[N: M]$ will be a prime ideal.

Claim $2:[x: M] M=[x: M]^{2} M$. If $[x: M] M \neq[x: M]^{2} M$, then $x \notin[x: M]^{2} M$, so $x \neq \alpha x$ for all $\alpha \in[x: M]$. Since $\alpha x=0$ or $\alpha x \in V(\bar{\Gamma}(M))$, we have $\alpha x$ adjacent to $x$. Therefore $\alpha^{2} \in[N: M]$. We know that $N$ is a prime submodule, so $\alpha \in[N: M]$ for all $\alpha \in[x: M]$. Thus $[x: M] M \subseteq N$, which is a contradiction with $x \notin \operatorname{Ann}(x) M$. Therefore $[x: M] M=[x: M]^{2} M$.

Claim $3: M=R x \oplus M_{2}$. Since $[x: M] M=[x: M]^{2} M$, we have $R x=$ $[x: M] x$. We know that $R x$ is a weak-cancellation $R$-module, and so $R=$ 
$[x: M]+\operatorname{Ann}(x)$. A simple check yields $M=R x \oplus \operatorname{Ann}(x) M$. Hence we may assume that $M=R x \oplus M_{2}$ with $x$ adjacent to every other vertex and $M_{2}=\operatorname{Ann}(x) M$.

Claim 4:Rx $=\{0, x\}$. Let $x \neq c \in R x$. Then $c \in V(\bar{\Gamma}(M))$ and $[c: M][x$ : $M] M=0$; hence $[c: M] x=0$, so $c \in R x \cap M_{2}=\{0\}$.

Claim $5: D\left(M_{2}\right)=0$. Let $D\left(M_{2}\right) \neq 0$. Then there exists a non-zero element $m_{2} \in M_{2}$ such that $\left[m_{2}: M\right]\left[m_{2}^{\prime}: M\right] M=0$ for some $0 \neq m_{2}^{\prime} \in M_{2}$. Thus $x+m_{2}^{\prime}$ is a vertex of $\bar{\Gamma}(M)$, which is adjacent to $x$. Therefore $[x: M]\left(x+m_{2}^{\prime}\right)=$ 0 , so

$$
[x: M] x=[x: M] m_{2}^{\prime} .
$$

Thus $[x: M] x \subseteq R x \cap M_{2}=\{0\}$. Hence $x \in \operatorname{Ann}(x) M$, which is a contradiction; consequently, $D\left(M_{2}\right)=0$.

Claim 6: $M_{2}=\{0, y\}$. Since $D\left(M_{2}\right)=0$, we have $[y: M] y \neq 0$. On the other hand, $0 \neq x \in \operatorname{Ann}(y) M$, so $y \in V(\bar{\Gamma}(M))$. From the above argument we have $[y: M]^{2} M=[y: M] M$. Therefore

$$
R y \subseteq[y: M] y \subseteq\left(\operatorname{Ann}(x) \cap\left[y: M_{2}\right]\right) y \subseteq\left[y: M_{2}\right] y .
$$

Hence $R y=\left[y: M_{2}\right] y$ and $y=s y$ for some $s \in\left[y: M_{2}\right]$. Let $m_{2} \in M_{2}$, so

$$
[y: M]\left[(1-s) m_{2}: M\right] M=0 .
$$

Thus $y=0$ or $m_{2}=s m_{2} \in R y$. Hence $M_{2}=R y$. Let $m_{2} \in M_{2}$ and $m_{2} \neq y$, so $m_{2} \in V(\bar{\Gamma}(M))$ and $\left[m_{2}: M\right][y: M] M=0$. Therefore $m_{2}=0$ and $R y$ has exactly two elements. Consequently, $|M|=4$.

Next, we may assume that $[x: M]^{2} M=0$ for all $x \in V(\bar{\Gamma}(M))$. So $x \in$ $\operatorname{Nil}(M)$ and $V(\bar{\Gamma}(M)) \subseteq \operatorname{Nil}(M)$. Now let $0 \neq x \in \operatorname{Nil}(M)$. We can write $x=\sum_{i=1}^{n} \alpha_{i} m_{i}$, where $\alpha_{i} \in[x: M], m_{i} \in M$ such that $\alpha_{i} m_{i} \neq 0$ for $1 \leq i \leq n$. On the other hand, $\alpha_{i}^{2} m_{i}=0$, so $0 \neq \alpha_{i} m_{i} \in \operatorname{Ann}\left(\alpha_{i} m_{i}\right) M$. Therefore $\alpha_{i} m_{i} \in$ $V(\bar{\Gamma}(M))$. One can easily check that $V(\bar{\Gamma}(M))$ is a submodule of $M$; hence $x \subseteq V(\bar{\Gamma}(M))$. Consequently, $\operatorname{Nil}(M)=V(\bar{\Gamma}(M)) \cup\{0\}$.

Example 2.10. Let $R=\mathbb{Z}$ and $M=\mathbb{Z}_{p^{2}}$, where $p>2$ is a prime number. It is clear that $\bar{\Gamma}(M)=K_{p-1}$ is a complete graph. So by Theorem $2.9, \operatorname{Nil}(M)=$ $(\bar{p})$.

\section{Isomorphisms}

Recall that two graphs $G$ and $H$ are isomorphic, denoted by $G \cong H$, whenever there exists a bijection, say $\varphi$ from $V(G)$ to $V(H)$, of vertices such that the vertices $x$ and $y$ are adjacent in $G$ if and only if $\varphi(x)$ and $\varphi(y)$ are adjacent in $H$.

Let $S=R \backslash Z(M)$. It is clear that the well-defined map

$$
\chi: M \longrightarrow S^{-1} M,
$$

defined by

$$
\chi(m)=\frac{m s}{s}
$$


is a monomorphism. So we can identify $M$ with its image in $S^{-1} M$. Thus if $m$ denotes an element of $M$, then the same symbol is also used to denote the fraction $\frac{m}{1}$. In this manner, $M$ become a submodule of $S^{-1} M$.

Let $M$ be an $R$-module. For $m, m^{\prime} \in V(\bar{\Gamma}(M))$, we define $m \sim_{M} m^{\prime}$ if and only if $\operatorname{Ann}(m)=\operatorname{Ann}\left(m^{\prime}\right)$. Clearly $\sim_{M}$ is an equivalence relation on $V(\bar{\Gamma}(M))$. Let $S=R \backslash Z(M)$. For $m \in M$, denote the equivalence classes of $\sim_{M}$ and $\sim_{M_{S}}$ containing $m, \frac{m}{1}$ by $[m]_{M}$ and $\left[\frac{m}{1}\right]_{M_{S}}$, respectively, so

$$
[m]_{M}=\left\{m^{\prime} \in V(\bar{\Gamma}(M)) \mid m \sim_{M} m^{\prime}\right\}
$$

and let

$$
\left[\frac{m}{1}\right]_{M_{S}}=\left\{\frac{m^{\prime}}{t^{\prime}} \in V\left(\bar{\Gamma}\left(M_{S}\right)\right) \mid \frac{m^{\prime}}{t^{\prime}} \sim_{M_{S}} \frac{m}{1}\right\} .
$$

Next, we prove that $\bar{\Gamma}\left(S^{-1} M\right)$ and $\bar{\Gamma}(M)$ are isomorphic by showing that there is a bijection map between equivalence classes of vertex sets $\bar{\Gamma}\left(S^{-1} M\right)$ and $\bar{\Gamma}(M)$ such that the corresponding equivalence classes have the same cardinality.

Theorem 3.1. Let $M$ be an $R$-module such that $\operatorname{Ann}(x)=\operatorname{Ann}([x: M] M)$ for all $x \in T(M)$ and $S=R \backslash Z(M)$. Then $\bar{\Gamma}(M)$ and $\bar{\Gamma}\left(S^{-1} M\right)$ are isomorphic.

Proof. (Our proof is quite similar to the proof in [7] applied for a ring) Let $S=R \backslash Z(M), M_{S}=S^{-1} M, R_{S}=S^{-1} R$. A simple check yields that for all $N \leq M$, we have $S^{-1} \operatorname{Ann}_{R}(N)=\operatorname{Ann}_{S^{-1} R}\left(S^{-1} N\right)$. Hence

$$
V\left(\bar{\Gamma}\left(M_{S}\right)\right)=\left\{\frac{m}{s} \mid m \in V(\bar{\Gamma}(M)), s \in S\right\},
$$

and $\left([m]_{M}\right)_{S}=\left(\left[\frac{m}{1}\right]\right)_{M_{S}}$. On the other hand,

$$
V(\bar{\Gamma}(M))=\bigcup_{\lambda \in \Lambda}\left[m_{\lambda}\right]_{M} \text {, so } V\left(\bar{\Gamma}\left(M_{S}\right)\right)=\bigcup_{\lambda \in \Lambda}\left[\frac{m_{\lambda}}{1}\right]_{M_{S}}
$$

(both are disjoint unions). Next we show that $\left|[x]_{M}\right|=\left|\left[\frac{x}{1}\right]_{M_{S}}\right|$ for all $x \in$ $V(\bar{\Gamma}(M))$. It is clear that $[x]_{M} \subseteq\left[\frac{x}{1}\right]_{M_{S}}$. For the reverse inclusion, assume $\frac{m}{s} \in\left[\frac{x}{1}\right]_{M_{S}}$. We can suppose that $m \in[x]_{M}, s \in S$, so $\operatorname{Ann}(m)=\operatorname{Ann}(x)$. Therefore $\left\{s^{n} m \mid n \geq 1\right\} \subseteq[x]_{M}$. If $\left|[x]_{M}\right|$ is finite, then there exists $i \in I$ such that $s^{i} m=s^{i+1} m$. So

$$
\frac{m}{s}=\frac{m s^{i}}{s^{i+1}}=\frac{m s^{i+1}}{s^{i+1}}=m \in[x]_{M} ;
$$

therefore $\left|[x]_{M}\right|=\left|\left[\frac{x}{1}\right]_{M_{S}}\right|$. Now suppose that $\left|[x]_{M}\right|$ is infinite. We define an equivalence relation $\approx$ on $S$ by $s \approx t$ if and only if $s x=t x$. It is easily verified that the map

$$
\begin{gathered}
{[x]_{M} \times S / \approx \longrightarrow\left[\frac{x}{1}\right]_{M_{S}}} \\
(b,[s]) \longrightarrow \frac{b}{s},
\end{gathered}
$$

is well-defined, because if $(b,[s])=(a,[t])$, then $a=b$ and $[s]=[t]$. Hence

$$
(s-t) M \subseteq \operatorname{Ann}(x) M=\operatorname{Ann}(a) M=\operatorname{Ann}(b) M ;
$$


by the hypothesis $s a=t a$ and $s b=t b$, therefore $\frac{a}{t}=\frac{b}{s}$. Also, it is clear that this map is surjective. Thus

$$
\left|\left[\frac{x}{1}\right]\right| \leq\left|[x]_{M}\right||S / \approx| .
$$

The map

$$
\begin{gathered}
S / \approx \longrightarrow[x]_{M} \\
{[s] \longrightarrow s a .}
\end{gathered}
$$

Clearly, it is well-defined and injective. Hence $|S / \approx| \leq\left|[x]_{M}\right|$, and

$$
\left|\left[\frac{x}{1}\right]_{M_{S}}\right| \leq\left|[x]_{M}\right|^{2}=\left|[x]_{M}\right|,
$$

since $\left|[x]_{M}\right|$ is infinite, $\left|[x]_{M}\right|=\left|\left[\frac{x}{1}\right]_{M_{S}}\right|$. Thus there is a bijection map

$$
\varphi_{\alpha}:\left[x_{\alpha}\right] \longrightarrow\left[\frac{x_{\alpha}}{1}\right]
$$

for each $\alpha \in \Lambda$. Therefore we define

$$
\varphi: V(\bar{\Gamma}(M)) \longrightarrow V\left(\bar{\Gamma}\left(M_{S}\right)\right)
$$

by $\varphi(m)=\varphi_{\alpha}(m)$, if $m \in\left[x_{\alpha}\right]_{M}$. Clearly, $\varphi$ is a bijection map. Thus we need only to show that $m$ and $n$ are adjacent in $\Gamma(M)$ if and only if $\varphi(m)$ and $\varphi(n)$ are adjacent in $\Gamma\left(M_{S}\right)$; that is, $[m: M][n: M] M=0$ if and only if $\left[\varphi(m): M_{S}\right]\left[\varphi(n): M_{S}\right] M_{S}=0$. Let $m \in[x]_{M}, n \in[y]_{M}, w \in\left[\frac{x}{1}\right]_{M_{S}}$, and $z \in\left[\frac{y}{1}\right]_{M_{S}}$. It is sufficient to show that $[m: M][n: M] M=0$ if and only if $\left[w: M_{S}\right]\left[z: M_{S}\right] M_{S}=0$. If $m$ is adjacent to $n$, then

$$
\begin{aligned}
& {[m: M][n: M] M=0 } \\
\Longrightarrow & {[m: M] \subseteq \operatorname{Ann}_{R}(n)=\operatorname{Ann}_{R}(y) } \\
\Longrightarrow & {[m: M]_{S} \subseteq \operatorname{Ann}_{R_{S}}\left(\frac{y}{1}\right)=\operatorname{Ann}_{R_{S}}(z) } \\
\Longrightarrow & {\left[z: M_{S}\right] \subseteq \operatorname{Ann}_{R_{S}}\left(([m: M] M)_{S}\right) } \\
\Longrightarrow & {\left[z: M_{S}\right] \subseteq \operatorname{Ann}\left(\frac{m}{1}\right)=\operatorname{Ann}\left(\frac{x}{1}\right)=\operatorname{Ann}(w) } \\
\Longrightarrow & {\left[z: M_{S}\right]\left[w: M_{S}\right] M_{S}=0 . }
\end{aligned}
$$

Conversely, if $z$ is adjacent to $w$, then

$$
\begin{aligned}
& {\left[z: M_{S}\right]\left[w: M_{S}\right] M_{S}=0 } \\
\Longrightarrow & {\left[z: M_{S}\right] \subseteq \operatorname{Ann}_{R_{S}}\left(\left[w: M_{S}\right] M_{S}\right) \subseteq \operatorname{Ann}_{R_{S}}(w) } \\
\Longrightarrow & {\left[z: M_{S}\right] \subseteq \operatorname{Ann}_{R_{S}}(w)=\operatorname{Ann}_{R_{S}}\left(\frac{x}{1}\right)=\operatorname{Ann}_{R_{S}}\left(\frac{m}{1}\right) } \\
\Longrightarrow & {\left[z: M_{S}\right]\left[\frac{x}{1}: M_{S}\right] M_{S}=0, }
\end{aligned}
$$

implies that

$$
\begin{aligned}
& {\left[\frac{m}{1}: M_{S}\right] \subseteq \operatorname{Ann}_{R_{S}}\left(\left[z: M_{S}\right] M_{S}\right) \subseteq \operatorname{Ann}_{R_{S}}(z)=\operatorname{Ann}_{R_{S}}\left(\frac{y}{1}\right)=\operatorname{Ann}_{R_{S}}\left(\frac{n}{1}\right) } \\
\Longrightarrow & {\left[\frac{n}{1}: M_{S}\right]\left[\frac{m}{1}: M_{S}\right] M_{S}=0 } \\
\Longrightarrow & {[m: M][n: M] M=0 }
\end{aligned}
$$

hence $\bar{\Gamma}(M)$ and $\bar{\Gamma}\left(M_{S}\right)$ are isomorphic graphs. 
Theorem 3.2. Let $M$ be a multiplication $R$-module and $S=R \backslash Z(M)$. Then $\Gamma(M)$ and $\Gamma\left(S^{-1} M\right)$ are isomorphic.

Proof. It is similar to the proof of Theorem 3.1.

Corollary 3.3. Let $M$ and $N$ be multiplication R-modules with $S^{-1} M \cong$ $S^{-1} N$. Then $\Gamma(M) \cong \Gamma(N)$. In particular, $\Gamma(M) \cong \Gamma(N)$ when $S^{-1} M=$ $S^{-1} N$.

\section{Complemented graph $\bar{\Gamma}(M)$ and multiplication module}

In this section we prove that, if $M$ is a reduced multiplication $R$-module and $\bar{\Gamma}(M)$ is uniquely complemented, $S^{-1} M$ is von Neumann regular. Furthermore, we show that if $M$ is a multiplication $R$-module with $\operatorname{Nil}(M) \neq 0$, then $\Gamma(M)$ is uniquely complemented if and only if $\bar{\Gamma}(M)$ is a star graph such that $\bar{\Gamma}(M)$ has at most six edges or is an infinite star graph. Finally, we show that if $M$ is a multiplication $R$-module, and $\bar{\Gamma}(M)$ is uniquely complemented, then either $\bar{\Gamma}(M)$ is a star graph or $S^{-1} M$ is von Neumann regular, where $S=R \backslash Z(M)$.

Let $G$ be a (undirected) graph. We will follow the authors in [6] and define that $a \leq b$ if $a$ and $b$ are not adjacent and each vertex of $G$ adjacent to $b$ is also adjacent to $a$; we define $a \sim b$ if and only if $a \leq b$ and $b \leq a$. Thus $a \sim b$ if and only if $a$ and $b$ are adjacent to exactly the same vertices. Clearly $\sim$ is an equivalence relation on $G$. Let $M$ be a multiplication $R$ module and $m, n \in T(M)^{*}$; then $m \sim n$ if and only if $\operatorname{Ann}(m) M \backslash\{m\}=$ $\operatorname{Ann}(n) M \backslash\{n\}$. We also know that if $m \perp n$, then $[m: M][n: M] M=0$ and $\operatorname{Ann}(m) M \cap \operatorname{Ann}(n) M \subseteq\{0, m, n\}$. Now if $\operatorname{Ann}(m) M \cap \operatorname{Ann}(n) M=\{0, m, n\}$, then $[m: M]^{2} M=[n: M]^{2} M=[m: M][n: M] M=0$ and so $m+n$ is adjacent to $m$ and $n$, since $m \perp n, m+n \in\{0, m, n\}$, which is a contradiction. Therefore $m \perp n$ if and only if $\operatorname{Ann}(m) M \cap \operatorname{Ann}(n) M \subset\{0, m, n\}$ and $[m$ : $M][n: M] M=0$.

Proposition 4.1. Let $M$ be a multiplication R-module. Then $M$ is von Neumann regular if and only if every cyclic submodule of $M$ is pure in $M$.

Proof. Let every cyclic submodule of $M$ be pure in $M$. Hence $R m=[m: M] m$ for all $m \in M$ and so $m=\alpha m$ for some $\alpha \in[m: M]$. Therefore, $M=$ $\operatorname{Ann}(m) M+R m$ so that $1 \in \operatorname{Ann}(m)+[m: M]$. On the other hand, if $x \in$ $\operatorname{Ann}(m) M \cap R m$, then $x=s m=r m_{0}$ for some $r \in \operatorname{Ann}(m)$ and $s \in R$. Thus $\alpha x=s \alpha m=r \alpha m_{0}=0$, so $x=0$. This implies that $M=\operatorname{Ann}(m) M \oplus R m$. Thus $M$ is von Neumann regular. The converse is obvious.

Lemma 4.2. Consider the following statements for a multiplication $R$-module $M$ with $m, m^{\prime} \in T(M)^{*}$.

(a) $m \sim m^{\prime}$

(b) $R m=R m^{\prime}$,

(c) $\operatorname{Ann}(m) M=\operatorname{Ann}\left(m^{\prime}\right) M$.

Then under the above conditions, we have: 
(1) If $M$ is reduced, then statements (a) and (c) are equivalent.

(2) If $M$ is von Neumann regular, then all three statements are equivalent.

Proof. (1) Let $M$ be reduced; one can easily check that (a) $\Longleftrightarrow$ (c).

(2) $(\mathrm{a}) \Longleftrightarrow(\mathrm{c})$; since every von Neumann regular module is reduced.

(b) $\Longrightarrow(\mathrm{c})$; this implication is clear.

$(\mathrm{c}) \Longrightarrow(\mathrm{b})$; Since $M$ is von Neumann regular, $R m \cap[m: M] M=[m: M] R m$. So $m=s m$ for some $s \in[m: M]$, hence; $(1-s) m^{\prime} \in \operatorname{Ann}(m) M=\operatorname{Ann}\left(m^{\prime}\right) M$. Therefore $\left[m^{\prime}: M\right] m^{\prime} \in R m$. Moreover, since $M$ is a von Neumann regular multiplication, module $\left[m^{\prime}: M\right] m^{\prime}=R m^{\prime}$. So $R m^{\prime} \subseteq R m$ and, similarly, $R m \subseteq R m^{\prime}$; consequently, $R m=R m^{\prime}$.

Lemma 4.3. Let $M$ be a reduced multiplication $R$-module with $m, m^{\prime}, m^{\prime \prime} \in$ $V(\bar{\Gamma}(M))$. If $m \perp m^{\prime}$ and $m \perp m^{\prime \prime}$, then $m^{\prime} \sim m^{\prime \prime}$. Thus $\bar{\Gamma}(M)$ is uniquely complemented if and only if $\bar{\Gamma}(M)$ is complemented.

Proof. Let $m, m^{\prime}, m^{\prime \prime} \in \bar{\Gamma}(M)$. Suppose $m \perp m^{\prime}$ and $m \perp m^{\prime \prime}$. It is sufficient to show that $\operatorname{Ann}\left(m^{\prime}\right) M=\operatorname{Ann}\left(m^{\prime \prime}\right) M$. Suppose $x \in \operatorname{Ann}\left(m^{\prime}\right) M$, so $[x: M]\left[m^{\prime}\right.$ : $M] M=0$. One can easily show that for all $\alpha \in[x: M]$,

$$
\left[\alpha m^{\prime \prime}: M\right]\left[m^{\prime}: M\right] M=0=\left[\alpha m^{\prime \prime}: M\right][m: M] M .
$$

So $\alpha m^{\prime \prime} \in\left\{0, m, m^{\prime}\right\}$. If $\alpha m^{\prime \prime}=m$ or $\alpha m^{\prime \prime}=m^{\prime}$, then $m=0$ or $m^{\prime}=0$ is a contradiction. Thus $\alpha m^{\prime \prime}=0$ for all $\alpha \in[x: M]$, and therefore $x \in \operatorname{Ann}\left(m^{\prime \prime}\right) M$ and $\operatorname{Ann}\left(m^{\prime}\right) M \subseteq \operatorname{Ann}\left(m^{\prime \prime}\right) M$. Similarly, $\operatorname{Ann}\left(m^{\prime \prime}\right) M \subseteq \operatorname{Ann}\left(m^{\prime}\right) M$.

As an immediate consequence, we obtain the following result.

Corollary 4.4. Let $M$ be a reduced multiplication $R$-module with $m, m^{\prime}, m^{\prime \prime} \in$ $T(M)^{*}$. If $m \perp m^{\prime}$ and $m \perp m^{\prime \prime}$, then $m^{\prime} \sim m^{\prime \prime}$. Thus $\Gamma(M)$ is uniquely complemented if and only if $\Gamma(M)$ is complemented.

Theorem 4.5. Let $R$ be a Bézout ring and $M$ be a reduced multiplication $R$ module. If $\bar{\Gamma}(M)$ is complemented, then $S^{-1} M$ is von Neumann regular, where $S=R \backslash Z(M)$.

Proof. Let $0 \neq \frac{x}{s} \in S^{-1} M$, where $x \in M$ and $s \in S$. Let $x \notin V(\bar{\Gamma}(M))$ and $x=\sum_{i=1}^{n} \alpha_{i} m_{i} \in[x: M] M$, where $\alpha_{i} \in[x: M]$ and $m_{i} \in M$. Since $R$ is a Bézout ring $\sum_{i=1}^{n} R \alpha_{i}=R \alpha$ for some $\alpha \in R$. So $x=\alpha m$ for some $\alpha \in M$. If $\alpha \in Z(M)$, then $\alpha m_{0}=0$ for some non-zero element $m \in M$. So $\left[m_{0}: M\right][x: M] M=0$; hence $0 \neq m_{0} \subseteq \operatorname{Ann}(x) M=0$, which is a contradiction. Therefore $\alpha \in S=R \backslash Z(M)$. Thus one can easily check that

$$
S^{-1} R\left(\frac{x}{s}\right) \cap S^{-1} M\left(\frac{r}{t}\right)=S^{-1} R\left(\frac{r}{t} \frac{x}{s}\right) .
$$

Therefore by Proposition 4.1, $S^{-1} M$ is von Neumann regular.

Next we assume that $x \in V(\bar{\Gamma}(M))$. By the hypothesis there is $y \in V(\bar{\Gamma}(M))$ such that $x \perp y$. Hence $y \in \operatorname{Ann}(x) M$ and so $y=\sum_{i=1}^{m} \beta_{i} m_{i}, m_{i} \in M$ and $\beta_{i} \in \operatorname{Ann}(x)$. Let $R \beta=\sum_{i=1}^{m} R \beta_{i}$ for some $\beta \in R$, so $y=\beta m^{\prime}$ for some 
$m^{\prime} \in M$. We show that $\alpha+\beta \in S$. If $\alpha+\beta \in Z(M)$, then $(\alpha+\beta) m_{1}=0$ for some non-zero $m_{1} \in M$. So $\left[\alpha m_{1}: M\right][x: M] M=0=[y: M]\left[\alpha m_{1}: M\right] M$. Since $M$ is a reduced module $x \neq \alpha m_{1}$ and $y \neq \alpha m_{1}$. Thus $\alpha m_{1}=0$, and hence $\beta m_{1}=0$. So

$$
[x: M]\left[m_{1}: M\right] M=0=[y: M]\left[m_{1}: M\right] M .
$$

By a similar argument we have $m_{1}=0$, a contradiction. Therefore $\alpha+\beta \in S$ and $\frac{x}{s}=\frac{\alpha}{\alpha+\beta} \frac{x}{s}$. A simple check yields that

$$
S^{-1} R\left(\frac{x}{s}\right) \cap S^{-1} M\left(\frac{r}{t}\right)=S^{-1} R\left(\frac{r}{t} \frac{x}{s}\right) .
$$

Hence by Proposition 4.1, $S^{-1} M$ is von Neumann regular.

Lemma 4.6. Let $R$ be a von Neumann regular ring. Then every multiplication $R$ - module is reduced.

Proof. Lemma 2.5 of [19].

As an immediate consequence, we obtain the following result.

Corollary 4.7. Let $R$ be a von Neumann regular ring and $M$ be a multiplication $R$-module. If $\bar{\Gamma}(M)$ is complemented, then $S^{-1} M$ is von Neumann regular, where $S=R \backslash Z(M)$.

Corollary 4.8. Let $M$ be a reduced cyclic R-module. If $\bar{\Gamma}(M)$ is complemented, then $S^{-1} M$ is von Neumann regular, where $S=R \backslash Z(M)$.

Proof. It is similar to the proof of Theorem 4.5.

Example 4.9. Let $R=\mathbb{Z}$ and $M=\mathbb{Z}_{2} \oplus \mathbb{Z}_{3}$. Clearly $M$ is reduced, and by Example 2.7, $\bar{\Gamma}(M)$ is complemented. So by Corollary $4.8, S^{-1} M$ is von Neumann regular.

Lemma 4.10. Let $R=\mathbb{Z}$ and $M=\mathbb{Z}_{p q}$ where $p$ and $q$ are distinct prime numbers. Then $\bar{\Gamma}(M)$ is complete bipartite.

Proof. Let $x \in V(\bar{\Gamma}(M))$, so either $x=t p$ or $x=s q$ for some $s, q \in R$. Therefore $\bar{\Gamma}(M)$ may be partitioned into two disjoint vertex sets $A$ and $B$, where $A=\{t p \mid t \in R, t p<n\}$ and $B=\{s q \mid s \in R s q<n\}$, and so $\bar{\Gamma}(M)$ is a complete bipartite graph.

Corollary 4.11. Let $M$ be a cyclic reduced $R$-module. The following statements are equivalent:

(1) $S^{-1} M$ is von Neumann regular, where $S=R \backslash Z(M)$.

(2) $\bar{\Gamma}(M)$ is uniquely complemented.

(3) $\bar{\Gamma}(M)$ is complemented. 
Proof. (1) $\Longrightarrow(2)$. Let $M$ be a von Neumann regular $R$-module and $m \in$ $V(\bar{\Gamma}(M))$. So $[m: M] M \cap R m=R m[m: M]$. Since $R m$ is a weak-cancellation module, $R=[m: M]+\operatorname{Ann}(m)$. Say $M:=R x$ for some $x \in M$. Thus $R x=$ $R m+\operatorname{Ann}(m) x$ and therefore $x=r m+y$ for some $r \in R, y \in \operatorname{Ann}(m) x$. One can easily check that $y \in V(\bar{\Gamma}(M))$ and $y \perp m$, so $\bar{\Gamma}(M)$ is complemented. Since $M$ is a cyclic $R$-module, then $S^{-1} M$ is a cyclic $S^{-1} R$-module, and therefore by the above comments, $\bar{\Gamma}\left(S^{-1} M\right)$ is complemented. Moreover, by Theorem 3.2 $\bar{\Gamma}(M) \cong \bar{\Gamma}\left(S^{-1} M\right)$, so $\bar{\Gamma}(M)$ is complemented. Consequently, $\bar{\Gamma}(M)$ is uniquely complemented by Lemma 4.3 .

$(2) \Longrightarrow(3)$. This is true for any graph.

$(3) \Longrightarrow(1)$. By Corollary 4.8 .

Corollary 4.12. Let $R=\mathbb{Z}$ and $M=\mathbb{Z}_{p_{1} p_{2} \ldots p_{n}}$, where $p_{i}, 1 \leq i \leq n$ are distinct prime numbers. Then $\bar{\Gamma}(M)$ is uniquely complemented and $S^{-1} M$ is von Neumann regular.

Proof. Let $n=3$ and $x \in V(\bar{\Gamma}(M))$. So there exist $i, j, k \in\{1,2,3\}$ such that either $x=t_{i} p_{i}$, where $t_{i} \in \mathbb{Z}$ and $p_{j}$ is not divisible by $t_{i}$ for $i \neq j$, or $x=s_{i} p_{k} p_{j}$ where $s_{i} \in \mathbb{Z}$ and $p_{j}$ is not divisible by $s_{i}$ for $i \neq j, i \neq k$. A routine argument shows that $x=t_{i} p_{i} \perp p_{k} p_{j}$ and $x=s_{i} p_{k} p_{j} \perp p_{i}$ for distinct $i, j, k$. Therefore by a similar argument we can show that $\bar{\Gamma}(M)$ is complemented, and by Corollary $4.11 \bar{\Gamma}(M)$ is uniquely complemented and $S^{-1} M$ is von Neumann regular.

The next example shows that $S^{-1} M$ is von Neumann regular, while $M$ is not von Neumann regular in spite of $\Gamma(M) \cong \Gamma\left(S^{-1} M\right)$.

Example 4.13. (a) Let $M_{1}$ be an $R_{1}$-module and $M_{2}$ an $R_{2}$-module; then $M=M_{1} \times M_{2}$ is an $R=R_{1} \times R_{2}$ module with this multiplication $R \times M \longrightarrow M$, defined by $\left(r_{1}, r_{2}\right)\left(m_{1}, m_{2}\right)=\left(r_{1} m_{1}, r_{2} m_{2}\right)$.

Now let $M=\mathbb{Z} \times n \mathbb{Z}$ and $R=\mathbb{Z} \times \mathbb{Z}$. Therefore the graph $\Gamma(M)$ is a complete bipartite graph (that is, $\Gamma(M)$ may be partitioned into two disjoint vertex sets, $V_{1}=\left\{\left(m_{1}, 0\right) \mid m_{1} \in(\mathbb{Z})^{*}\right\}$ and $V_{2}=\left\{\left(0, m_{2}\right) \mid m_{2} \in(n \mathbb{Z})^{*}\right\}$, and two vertices $x$ and $y$ are adjacent if and only if they are in distinct vertex sets). Therefore $\Gamma(M)$ is complemented. Also, $M$ is a faithful multiplication $R$-module, since $M=R(1, n)$. A simple check yields that $M$ is reduced. Thus $S^{-1} M$ is von Neumann regular by Corollary 4.8. But $M$ is not von Neumann regular (use $N=R(2,2 n)$ and $I=[N: M]$ ).

(b) Let $R=\mathbb{Z}_{2} \times \mathbb{Z}$ and $M=R$ as an $R$-module. So $M$ is a faithful multiplication $R$-module. Clearly, $M$ is reduced and $\Gamma(M)$ is an infinite star graph with center $(\overline{1}, 0)$. Thus $\Gamma(M)$ is complemented; by Corollary $4.8, S^{-1} M$ is von Neumann regular, but $M$ is not von Neumann regular.

Lemma 4.14. Let $M$ be a multiplication $R$-module; if $x \in N i l(M)$, then there exists $n \in \mathbb{N}$ such that $\alpha^{n} x=0$ for all $\alpha \in[x: M]$.

Proof. By the proof of Lemma 3.7 Step (1) of [16]. 
Proposition 4.15. Let $M$ be a multiplication $R$-module with $N i l(M) \neq 0$. Then

(a) If $\bar{\Gamma}(M)$ is complemented, then either $|M| \leq 16$ or $|M|>16$ and $\operatorname{Nil}(M)=\{0, x\}$ for some $0 \neq x \in M$.

(b) If $\bar{\Gamma}(M)$ is uniquely complemented with $|M|>16$, then any complement of the non-zero $x \in \operatorname{Nil}(M)$ is an end.

Proof. (a) We subdivide the proof of (a) into the following steps:

Let $\bar{\Gamma}(M)$ be complemented and $x \in \operatorname{Nil}(M)$. Assume that $\alpha \in[x: M]$, by Lemma $4.14 \alpha^{n} x=0$ for some $n \in \mathbb{N}$. Choose $n$ to be as small as possible, $\alpha^{n} x=0$. Then $n \geq 1$ and $\alpha^{n-1} x \neq 0$.

Step 1: In this step we claim that $n \leq 3$. Suppose that $n>3$, so $\alpha x \in$ $V(\bar{\Gamma}(M))$. Since $\bar{\Gamma}(M)$ is complemented, there is a $y \in V(\bar{\Gamma}(M)$ such that $y$ is a complement of $\alpha x$. Then

$$
\left[\alpha^{n-1} x: M\right][y: M] M=0=\left[\alpha^{n-1} x: M\right][\alpha x: M] M,
$$

so $\alpha^{n-1} x=y$ will be the only possibility. Thus $\alpha x \perp \alpha^{n-1} x$. Similarly, $\alpha^{i} x \perp \alpha^{n-1} x$ for each $1 \leq i \leq n-2$. Let $m=\alpha^{n-2} x+\alpha^{n-1} x$. Then

$$
[m: M]\left[\alpha^{n-1} x: M\right] M=0=[m: M]\left[\alpha^{n-2} x: M\right] M,
$$

which is a contradiction, since $\alpha^{n-2} x \perp \alpha^{n-1} x$ and $\alpha^{n-2} x+\alpha^{n-1} x \notin\left\{0, \alpha^{n-1} x\right.$, $\left.\alpha^{n-2} x\right\}$. Thus $n \leq 3$.

Step 2: Let $n=3$, so $\alpha^{3} x=0$ but $\alpha^{2} x \neq 0$. We show that $|M| \leq 16$. Similar to step 1, $\alpha x \perp \alpha^{2} x$. Also, $\operatorname{Ann}(x) M \subseteq\left\{0, \alpha^{2} x\right\}$, since if $z \in \operatorname{Ann}(x) M$, then $[z: M][x: M] M=0$; hence $z$ is adjacent to the two elements $\alpha x$ and $\alpha^{2} x$. Therefore $z=\alpha^{2} x$, so $\operatorname{Ann}(x) M \subseteq\left\{0, \alpha^{2} x\right\}$. In this case $R \alpha^{2} x=\left\{0, \alpha^{2} x\right\}$, because for all $r \in R$,

$$
\left[r \alpha^{2} x: M\right][\alpha x: M] M=0=\left[r \alpha^{2} x: M\right]\left[\alpha^{2} x: M\right] M ;
$$

hence $r \alpha^{2} x \in\left\{0, \alpha x, \alpha^{2} x\right\}$. But if $r \alpha^{2} x=\alpha x$, then $\alpha^{2} x=0$ is a contradiction, and so $R \alpha^{2} x=\left\{0, \alpha^{2} x\right\}$. Also,

$$
\operatorname{Ann}\left(\alpha^{2} x\right) M \subseteq\left\{0, x, \alpha x, \alpha^{2} x, x+\alpha x, x+\alpha^{2} x, \alpha x+\alpha^{2} x, x+\alpha x+\alpha^{2} x\right\},
$$

since if $z \in \operatorname{Ann}\left(\alpha^{2} x\right) M$, then $\alpha^{2} z \in \operatorname{Ann}(x) M=\left\{0, \alpha^{2} x\right\}$ and either $\alpha^{2} z=0$ or $\alpha^{2} z=\alpha^{2} x$. Thus either

$$
[\alpha z: M][\alpha x: M] M=0=[\alpha z: M]\left[\alpha^{2} x: M\right] M
$$

or

$$
[(\alpha z-\alpha x): M][\alpha x: M] M=0=[(\alpha z-\alpha x): M]\left[\alpha^{2} x: M\right] M .
$$

Since $\alpha x \perp \alpha^{2} x$, we have either $\alpha z \in\left\{0, \alpha x, \alpha^{2} x\right\}$ or $(\alpha z-\alpha x) \in\left\{0, \alpha x, \alpha^{2} x\right\}$. Now let $\alpha^{2} z=0$, so $\alpha z \neq \alpha x$; therefore either $\alpha z=0$ or $\alpha(z-\alpha x)=0$. So

$$
[z: M][\alpha x: M] M=0=[z: M]\left[\alpha^{2} x: M\right] M
$$

or

$$
[(z-\alpha x): M][\alpha x: M] M=0=[(z-\alpha x): M]\left[\alpha^{2} x: M\right] M
$$


hence $z \in\left\{0, \alpha x, \alpha^{2} x, \alpha^{2} x+\alpha x\right\}$. Thus we may assume that $\alpha^{2} z=\alpha^{2} x$; then $\alpha z-\alpha x \neq \alpha x$. On the other hand, $\alpha z-\alpha x \in\left\{0, \alpha x, \alpha^{2} x\right\}$, so either $\alpha z-\alpha x=0$ or $(\alpha z-\alpha x)=\alpha^{2} x$, and by a similar argument, $z \in\left\{x, \alpha^{2} x, x+\alpha x, x+\alpha x+\right.$ $\left.\alpha^{2} x\right\}$. Consequently,

$$
\operatorname{Ann}\left(\alpha^{2} x\right) M \subseteq\left\{0, x, \alpha x, \alpha^{2} x, x+\alpha x, x+\alpha^{2} x, \alpha x+\alpha^{2} x, x+\alpha x+\alpha^{2} x\right\} .
$$

Since $\alpha^{2}[x: M] M \neq 0$, there are $\gamma \in[x: M]$ and $m \in M$ such that $\alpha^{2} \gamma m \neq 0$, and a simple check yields $\alpha^{2} \gamma m=\alpha^{2} x$. Let $m_{0} \in M$, so $\alpha^{2} \gamma m_{0} \in R \alpha^{2} x=$ $\left\{0, \alpha^{2} x\right\}$. If $\alpha^{2} \gamma m_{0}=0$, then $m_{0} \in \operatorname{Ann}\left(\alpha^{2} x\right) M$, and if $\alpha^{2} \gamma m_{0}=\alpha^{2} x$, then $m_{0}-m \in \operatorname{Ann}\left(\alpha^{2} x\right)$. Consequently, $|M| \leq 16$.

Step 3: In this step we show that $H=\operatorname{Ann}\left(\alpha^{2} x\right) M$ is a unique maximal submodule of $\mathrm{M}$. Clearly, $H \neq M$ and $R \alpha^{2} x \cong \frac{R}{\operatorname{Ann}\left(\alpha^{2} x\right)}$. Since $R \alpha^{2} x=$ $\left\{0, \alpha^{2} x\right\}, \operatorname{Ann}\left(\alpha^{2} x\right)$ is a maximal ideal of $R$. Hence by Theorem 2.5 [15], $\operatorname{Ann}\left(\alpha^{2} x\right) M$ is a maximal submodule. Also,

$$
\operatorname{Ann}\left(\alpha^{2} x\right) M \subseteq R x \subseteq N i l(M) \subseteq \operatorname{Ann}\left(\alpha^{2} x\right) M .
$$

Therefore $\operatorname{Ann}\left(\alpha^{2} x\right) M=N i l(M)$ is a unique maximal submodule of $M$. Also, a simple check yields that $R m \neq M$ for all $m \in V(\bar{\Gamma}(M))$. Therefore by Theorem 2.5 [15] $R m \subseteq H$, so $V(\bar{\Gamma}(M)) \subseteq H$. So $V(\bar{\Gamma}(M))=\operatorname{Ann}\left(\alpha^{2} x\right) M$, so $\bar{\Gamma}(M)$ is a star graph with center $\alpha^{2} x$ and at most 6 edges.

Step (4): Assume that $n=2$; we show that $[x: M]^{2} x=0$. Let $[x: M]^{2} x \neq 0$. There exist two elements $\alpha, \beta \in[x: M]$ such that $\alpha \beta x \neq 0$. Also, $\alpha \beta \gamma m \neq 0$ for some $m \in M$ and $\gamma \in[x: M]$. On the other hand, $\alpha^{2} x=\beta^{2} x=\gamma^{2} x=0$ and $\alpha x \perp y$ for some $y \in V(\bar{\Gamma}(M))$. A simple check yields that $R \alpha x \subseteq$ $\{0, \alpha x, y\}$ and $y=\alpha \beta x$. Hence $\alpha x \perp \alpha \beta x$. So $R(\alpha x)=\{0, \alpha x, \alpha \beta x\}$ and $\operatorname{Ann}(\alpha x) M=\{0, \alpha x, \alpha \beta x\}$. Also, $\alpha \beta \gamma m$ is adjacent to two vertices $\alpha x$ and $\alpha \beta x$, but $\alpha \beta \gamma m \neq \alpha x$. Thus $\alpha \beta \gamma m=\alpha \beta x$. We know that $\alpha \beta m$ is adjacent to two vertices, $\alpha x$ and $\alpha \beta x$, but $\alpha \beta m \neq \alpha \beta \gamma m=\alpha \beta x$, so $\alpha \beta m=\alpha x$, which is a contradiction. Thus $[x: M]^{2} x=0$.

Step (5): Assume that $n=2$ and $[x: M]^{2} x=0$. We show that $|M| \leq 12$. By hypothesis, $\alpha^{2} x=0$ and $\alpha x \neq 0$; hence $\alpha[x: M] M \neq 0$. Thus $\alpha \beta m \neq 0$ for some $\beta \in[x: M]$ and $m \in M$. We know that $\Gamma(M)$ is complemented and $0 \neq \alpha \beta m \in \operatorname{Ann}(x) M$, so $x \in V(\bar{\Gamma}(M))$. So there is $y \in V(\bar{\Gamma}(M))$ such that $x \perp y$, but $\alpha x$ is adjacent to two vertices, $x$ and $y$. Hence either $\alpha x=x$ or $\alpha x=y$. If $\alpha x=x$, then multiplying by $\alpha$ we have $\alpha x=0$, which is a contradiction, so $\alpha x=y$. Let $z \in \operatorname{Ann}(x) M$. Hence $z \in\{0, x, \alpha x\}$, since $x \perp \alpha x=y$. If $z=x$, then $[x: M] x=0$, which is a contradiction. Therefore $\operatorname{Ann}(x) M=\{0, \alpha x\}$. Also, a simple check yields that $R(\alpha x)=\{0, \alpha x\}$. On the other hand, $\alpha \beta m \in \operatorname{Ann}(\alpha m) M$, so $\alpha m \in V(\bar{\Gamma}(M))$, and there exists $w \in V(\bar{\Gamma}(M))$ such that $\alpha m \perp w$; but $\alpha \beta m$ is adjacent to two vertices, $\alpha m$ and $w$. Therefore $\alpha \beta m=w$ will be the only possibility, and so $\alpha \beta m \perp \alpha m$. Also, $\alpha \beta m$ is adjacent to two vertices, $\alpha x$ and $x$; hence $\alpha \beta m=\alpha x$. Now we show that $\operatorname{Ann}(\alpha x) M=\{0, \alpha m, \alpha x, x, x+\alpha m, x+\alpha x\}$. Let $v \in \operatorname{Ann}(\alpha x) M$, 
so $\alpha v \in \operatorname{Ann}(x) M=\{0, \alpha x\}$. If $\alpha v=0$, then

$$
[v: M][\alpha \beta m: M] M=0=[v: M][\alpha m: M] M,
$$

and if $\alpha v=\alpha x$, then

$$
[v-x: M][\alpha \beta m: M] M=0=[v-x: M][\alpha m: M] M .
$$

Consequently, $\operatorname{Ann}(\alpha x) M=\{0, \alpha m, \alpha x, x, x+\alpha m, x+\alpha x\}$, and $|\operatorname{Ann}(\alpha x) M| \leq$ 6. For all $m_{0} \in M, \alpha \beta m_{0} \in R(\alpha x)=\{0, \alpha x\}$. So either $m_{0} \in \operatorname{Ann}(\alpha x) M$ or $m_{0}-m \in \operatorname{Ann}(\alpha x) M$, since $\alpha \beta m=\alpha x$. Therefore $|M| \leq 12$. By a similar argument in Step (3), $\operatorname{Ann}(\alpha x) M=\operatorname{Nil}(M)$ is a unique maximal submodule of $M$, and $\bar{\Gamma}(M)$ is a star graph with a center $\alpha x$ with at most 4 edges.

Step (6): Assume that $n=1$. If $[x: M] x \neq 0$, based on the above steps we have $6 \leq|M| \leq 16$. So we may assume that $[x: M] x=0$. We show that $|M|=9$ or $\operatorname{Nil}(M)=\{0, x\}$ with $2 x=0$ and $|M| \neq 9$. Let $x \in[x: M] M$ so $x=\sum_{i=1}^{n} \alpha_{i} m_{i}$ where $\alpha_{i} \in[x: M]$ and $m_{i} \in M$ for all $1 \leq i \leq n$. Since $\bar{\Gamma}(M)$ is complemented, there is $y \in T(M)^{*}$ such that $x \perp y$, so $R x \subseteq\{0, x, y\}$. If $x \neq \alpha_{i} m_{i}$ for all $i$, then $\alpha_{i} m_{i} \in R x$, and so $\alpha_{i} m_{i}=y$ for all $i$. Suppose that $\alpha_{i} m_{i}=\alpha_{1} m_{1}$; thus $x=\sum_{i=1}^{n} \alpha_{1} m_{1}=\left(\sum_{i=1}^{n} \alpha_{1}\right) m_{1}=\beta m_{1}$ where $\beta=\Sigma_{i=1}^{n} \alpha_{1} \in$ $[x: M]$. Hence we may assume that $x=\alpha m$ for some $\alpha \in[x: M]$ and $m \in M$ such that $\alpha^{2} m=0$, but $0 \neq \alpha m$. We know that $x+x \in R x \subseteq\{0, x, y\}$; if $x+x \neq 0$, then $R x=\{0, x, 2 x\}, x \perp 2 x$, and $\operatorname{Ann}(x) M=\{0, x, 2 x\}$. For all $m_{0} \in M, \alpha m_{0} \in R x$; therefore

$$
\begin{aligned}
{\left[m_{0}: M\right][x: M] M=0 } & =\left[m_{0}: M\right][2 x: M] \\
& \text { or } \\
{\left[m_{0}-m: M\right][x: M] M=0 } & =\left[m_{0}-m: M\right][2 x: M] \\
\text { or } & \\
{\left[m_{0}-2 m: M\right][x: M] M=0 } & =\left[m_{0}-2 m: M\right][2 x: M] .
\end{aligned}
$$

Hence $|M|=9$, and by a similar argument in Step (3), $\operatorname{Ann}(x) M$ is a unique maximal submodule of $M$ and $\bar{\Gamma}(M)$ is a star graph. Now let $|M| \neq 9$. So by the above argument, we must have $2 x=0$. We claim that $\operatorname{Nil}(M)=\{0, x\}$. Suppose that $z$ is another non-zero element of $N i l(M)$; hence $[z: M] z=0$ and $z=\beta m^{\prime}$ for some $\beta \in[z: M]$ and $m^{\prime} \in M$, such that $\beta^{2} m^{\prime}=0$. Also, $0 \neq x \in \operatorname{Ann}(x) M$ and $0 \neq z \in \operatorname{Ann}(z) M$, so $x, z \in V(\bar{\Gamma}(M))$. Since $\bar{\Gamma}(M)$ is complemented, there are $x^{\prime}, z^{\prime} \in V(\bar{\Gamma}(M))$ such that $x \perp x^{\prime}$ and $z \perp z^{\prime}$. Therefore $R x \subseteq\left\{0, x, x^{\prime}\right\}$ and $R z \subseteq\left\{0, z, z^{\prime}\right\}$. Observe that $\alpha \beta m=0$. Let $0 \neq \alpha \beta m \in R x$ and $\alpha \beta m \in R z$, if $\alpha \beta m=x \in R z$. Thus $x=z^{\prime}$, so $x \perp z$, and hence, $\alpha \beta m=0$ is a contradiction. If $\alpha \beta m=x^{\prime}$, then $R x=$ $\{0, x, \alpha \beta m\}=\operatorname{Ann}(x) M$, and, similar to the above argument, $|M|=9$, which is a contradiction. So $\alpha \beta m=0$. On the other hand, $x=\beta m^{\prime} \in \operatorname{Ann}(x+z) M$, so $x+z \in V(\bar{\Gamma}(M))$. Let $w$ be a complement of $x+z$; clearly, $w$ is neither $x$ nor $z$. It is clear that $\alpha w \in R x \subseteq\left\{0, x, x^{\prime}\right\}$, if $\alpha w=0$. Then $x$ is adjacent to two elements, $w$ and $x+z$, which is a contradiction. If $\alpha w=x^{\prime}$, then $R x=$ 
$\{0, x, \alpha w\}=\operatorname{Ann}(x) M$, and it implies that $|M|=9$, which is a contradiction. Hence we may assume that $\alpha w=x$ and similarly, $\beta w=z$. Then

$$
R z=[z: M] w, R x=[x: M] w .
$$

Since $w \perp x+z$,

$$
[w: M] x=[w: M] z,
$$

and $x, x+z \in R z$. Hence $x+z=z^{\prime}=x$ or $x+z=0$. In both case, we have a contradiction. Consequently, $\operatorname{Nil}(M)=\{0, x\}$.

(b) Let $0 \neq x \in \operatorname{Nil}(M)$ and $|M| \geq 17$. By the proof (a) we have $\operatorname{Nil}(M)=$ $\{0, x\}$ for some $x \in M$ such that $2 x=0$ and $[x: M] x=0$. Hence $x \in V(\bar{\Gamma}(M))$. Since $\bar{\Gamma}(M)$ is complemented, there is $y \in V(\bar{\Gamma}(M))$ such that $x \perp y$. We claim that $y$ is an end. We first show that $x+y$ also is a complement for $x$. Clearly, $x+y \in V(\bar{\Gamma}(M))$ and $[x+y: M][x: M] M=0$, because $[x: M] x=0$ and $x \perp y$. If $w \in V(\bar{\Gamma}(M))$ is adjacent to both $x$ and $x+y$, then

$$
[x+y: M][w: M] M=0=[x: M][w: M] M .
$$

Hence $[w: M] R(x+y)=0$, so $[y: M][w: M] M=0$. Moreover, $x \perp y$, thus either $w=x$ or $w=y$. If $w=y$, then $[y: M] y=0$. Therefore $y \in \operatorname{Nil}(M)=\{0, x\}$, which is a contradiction, so $x=w$. Thus $x+y$ is a complement for $x$. Since $\bar{\Gamma}(M)$ is uniquely complemented, $x+y \sim y$. Assume that $z \in V(\bar{\Gamma}(M)) \backslash\{x\}$ such that $z$ is adjacent to $y$; hence, $z$ is adjacent to $x+y$. So $[z: M][x: M] M=0$. Thus $z=y$, since $x \perp y$. Consequently, $y$ is an end.

Remark 4.16. The proof of Proposition 4.15(a) shows that if $M$ is a multiplication $R$-module such that $\bar{\Gamma}(M)$ is complemented and $|N i l(M)|>2$, then $|M| \leq 16$. Also, $M$ has a unique maximal submodule and $\bar{\Gamma}(M)$ is a star graph with at most six edges. Therefore $\bar{\Gamma}(M)$ is uniquely complemented. Also, it shows that if $\bar{\Gamma}(M)$ is not uniquely complemented, then $\operatorname{Nil}(M)=\{0, x\}$, in which $x$ is an element of $M$ such that $x[x: M]=0$. Hence $x=\beta m$ for some $m \in M$, and $\beta \in[x: M]$.

Example 4.17. Let $R=\mathbb{Z}$ and $M=\mathbb{Z}_{20}$. Clearly, $M$ is not reduced, so $\operatorname{Nil}(M) \neq 0$ and $\Gamma(M)$ is complemented, but not uniquely complemented. So by the proof of Proposition 4.15, Nil $(M)=\{0,10\}$.

Clearly, star graphs are uniquely complemented. The next theorem shows that for a multiplication $R$-module $M$ with $N i l(M) \neq 0$, if $\bar{\Gamma}(M)$ is uniquely complemented, then $\bar{\Gamma}(M)$ is a star graph.

Theorem 4.18. Let $R$ be a Bézout ring and $M$ be a multiplication $R$-module with $\operatorname{Nil}(M) \neq 0$. If $\bar{\Gamma}(M)$ is a uniquely complemented graph, then either $\bar{\Gamma}(M)$ is a star graph with at most six edges or $\bar{\Gamma}(M)$ is an infinite star graph with center $x$, where $\operatorname{Nil}(M)=\{0, x\}$. 


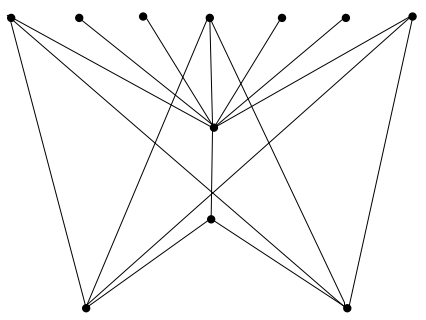

FIGURE 3

Proof. Suppose that $\bar{\Gamma}(M)$ is uniquely complemented and $\operatorname{Nil}(M) \neq 0$. Let $|M| \leq 16$; then by Remark 4.16, $\bar{\Gamma}(M)$ is a star graph with at most six edges. Let $|M|>16$. Hence by Step (7) of Proposition 4.15(a), $\operatorname{Nil}(M)=\{0, x\}$ for some $0 \neq x \in M$ and $[x: M] x=0$.

We first show that $\bar{\Gamma}(M)$ is an infinite graph. Let $c$ be a complement of $x$, so $\operatorname{Ann}(c) M=\{0, x\}=N i l(M)$, by Proposition $4.15(\mathrm{~b})$. Let $c=\Sigma_{i=1}^{n}\left(\alpha_{i} m_{i}\right) \in$ $[c: M] M$, where $\alpha_{i} \in[c: M]$ and $m_{i} \in M$ for $1 \leq i \leq n$. Since $R$ is a Bézout ring, $\sum_{i=1}^{n} R \alpha_{i}=R \alpha$ for some $\alpha \in R$. We claim that $\alpha c$ is also a complement of $x$. If $z$ is adjacent to both vertices $x$ and $\alpha c$, then

$$
[\alpha c: M][z: M] M=0=[x: M][z: M] M .
$$

Therefore $\alpha z \in \operatorname{Ann}(c) M=\{0, x\}$. So either $\alpha z=0$ or $\alpha z=x$. If $\alpha z=0$, then $[z: M] c=0$; so $z \in \operatorname{Ann}(c) M$, which is a contradiction, and $\alpha z=x$. Hence $\alpha[z: M] z=x[z: M]=0$. Therefore $z[z: M] \subseteq \operatorname{Ann}(c) M=\operatorname{Nil}(M)$, and hence $z \in \operatorname{Nil}(M)=\{0, x\}$, which again is a contradiction. Consequently, $\alpha c \perp x$; so by Proposition 4.15(b), $\operatorname{Ann}(\alpha c) M=\{0, x\}$. By a similar argument, $\alpha^{i} c \perp x$ and $\operatorname{Ann}\left(\alpha^{i} c\right) M=\{0, x\}$ for $1 \leq i \leq n$. Hence each $\alpha^{i} c$ is an end. Next, note that $\alpha^{i} c$ are all distinct. If not, suppose that $\alpha^{i} c=\alpha^{j} c$ for some $1 \leq i<j$. Therefore $\alpha^{i}\left(1-\alpha^{j-i}\right) c=0$, so $\left(1-\alpha^{j-i}\right) \in \operatorname{Ann}\left(\alpha^{i} c\right)$. Using the proof of Proposition 4.15(a) Step 6, $x=\beta m$ for some $\beta \in[x: M]$ and $m \in M$, such that $\beta^{2} m=0$ but $\beta m \neq 0$. Hence $\left(1-\alpha^{j-i}\right) m \in \operatorname{Ann}\left(\alpha^{i} c\right) M=\{0, x\}$. So either $m-\alpha^{i-j} m=0$ or $m-\alpha^{i-j} m=x$. If $m=\alpha^{i-j} m$, then

$$
x=\beta m=\beta \alpha^{i-j} m \in \beta \alpha^{i-j-1} R c \subseteq \alpha^{i-j-1}[x: M][c: M] M=0,
$$

which is a contradiction. Thus $m-\alpha^{i-j} m=x$. So

$$
x-\alpha^{i-j} \beta m=\beta m-\alpha^{i-j} \beta m=\beta x=0 .
$$

Hence $x \in \alpha^{i-j-1} \beta R c=0$, which again is a contradiction. Consequently, $\bar{\Gamma}(M)$ is infinite.

Next, we show that $\bar{\Gamma}(M)$ is a star graph with center $x$. By contradiction, suppose that $\bar{\Gamma}(M)$ is not a star graph. Let $c \in V(\bar{\Gamma}(M))$ be a complement of $x$, so there is a $a \in V(\bar{\Gamma}(M)) \backslash\{x, c\}$ such that $[a: M][x: M] M=0$, but $a$ is not an end. Hence there is $y \in V(\bar{\Gamma}(M)) \backslash\{a, x, c\}$ such that $y \perp a$. Let $c=\sum_{i=1}^{n}\left(\alpha_{i} m_{i}\right)$, where $\alpha_{i} \in[c: M]$ and $m_{i} \in M$, for $1 \leq i \leq n$, and 
let $R \alpha=\Sigma_{i=1}^{n} R \alpha_{i}$. We can check that $\alpha y \notin\{0, a, x, c, y\}$. If $\alpha y=0$, then $[y: M] c=0$, which is a contradiction with $c$ is an end. If $\alpha y=x$, then $\alpha[y: M][c: M] M=0$, so $y \in \operatorname{Ann}(\alpha c) M=\{0, x\}$, which is a contradiction. If $\alpha y=y$, then $\alpha y[x: M] \subseteq[x: M] R c=0$, which is a contradiction. If $\alpha y=c$, then $a$ is adjacent to $c$, which is a contradiction. Last, if $\alpha y=a$, then $\alpha y[y$ : $M]=0$. So $y[y: M] \in \operatorname{Ann}(\alpha c) M=N i l(M)$, and therefore $y \in N i l(M)$, which is a contradiction. Thus $\alpha y \in V(\bar{\Gamma}(M)) \backslash\{a, x, c, y\}$. By the hypothesis, there is $z \in V(\bar{\Gamma}(M))$ such that $z$ is a complement of $\alpha y$. One can also verify that $z \notin\{0, \alpha y, a, x, c, y\}$ (Use $y \notin N i l(M)$ to show that $z \notin\{c, y\}$ and use $\alpha y \perp z$ to show that $z \notin\{a, x\})$. Clearly, $[x: M][z: M] M \neq 0$. Let $z=\Sigma_{i=1}^{s} r_{i} m_{i}$, where $r_{i} \in[z: M]$ and $m_{i} \in M$ for $1 \leq i \leq s$, and let $R \gamma=\sum_{i=1}^{n} R r_{i}$. If $\gamma x=0$, then $[x: M][z: M] M=0$, which is a contradiction. So we must suppose that $\gamma x \neq 0$. Also, $[\gamma x: M][c: M] M=0$; hence $\gamma x \in \operatorname{Ann}(c) M$. Thus $\gamma x=x$. On the other hand, $\alpha y \perp z$, so

$$
[\gamma y: M][c: M] M=[y: M] R\left(\sum_{i=1}^{n}\left(\gamma \alpha_{i} m_{i}\right)\right) \subseteq[y: M] R \alpha z=0 .
$$

Therefore $\gamma y \in \operatorname{Ann}(c) M$. Hence either $\gamma y=0$ or $\gamma y=x$. So $x$ is adjacent to both $y$ and $a$, but this is a contradiction with $a \perp y$; consequently, $\bar{\Gamma}(M)$ is an infinite star graph with center $x$.

Corollary 4.19. Let $M$ be a cyclic $R$-module with $\operatorname{Nil}(M) \neq 0$. If $\bar{\Gamma}(M)$ is a uniquely complemented graph, then either $\bar{\Gamma}(M)$ is a star graph with at most six edges or $\bar{\Gamma}(M)$ is an infinite star graph with center $x$, where $\operatorname{Nil}(M)=\{0, x\}$.

Proof. It is similar to the proof of Theorem 4.18.

Corollary 4.20. Let $R$ be a Bézout ring and $M$ be a multiplication $R$ module. If $\bar{\Gamma}(M)$ is uniquely complemented, then either $\bar{\Gamma}(M)$ is a star graph or $S^{-1} M$ is von Neumann regular. Moveover, for a cyclic $R$-module $M$, the converse is true.

Proof. Let $\bar{\Gamma}(M)$ be uniquely complemented. If $N i l(M)=0$, then $M$ is reduced and by Theorem 4.5, $S^{-1} M$ is von Neumann regular. If $\operatorname{Nil}(M) \neq 0$, then by Theorem 4.18, $\bar{\Gamma}(M)$ is a star graph. The converse is true by Corollary 4.11.

Corollary 4.21. Let $M$ be a cyclic $R$ module. Then $\bar{\Gamma}(M)$ is uniquely complemented if and only if either $\bar{\Gamma}(M)$ is a star graph or $S^{-1} M$ is von Neumann regular.

Proof. Let $\bar{\Gamma}(M)$ be uniquely complemented. If $N i l(M)=0$, then $M$ is reduced, and by Corollary $4.8, S^{-1} M$ is von Neumann regular. If $\operatorname{Nil}(M) \neq 0$, then by Corollary 4.19, $\bar{\Gamma}(M)$ is a star graph. The converse is true by Corollary 4.11 .

Example 4.22. Let $R=\mathbb{Z}, M_{1}=\mathbb{Z}_{33}$, and $M_{2}=\mathbb{Z}_{30} \cdot \Gamma\left(M_{i}\right), i=1,2$, is a uniquely complemented graph. So by Corollary $4.20, S^{-1} M_{i}, i=1,2$, is von Neumann regular. 


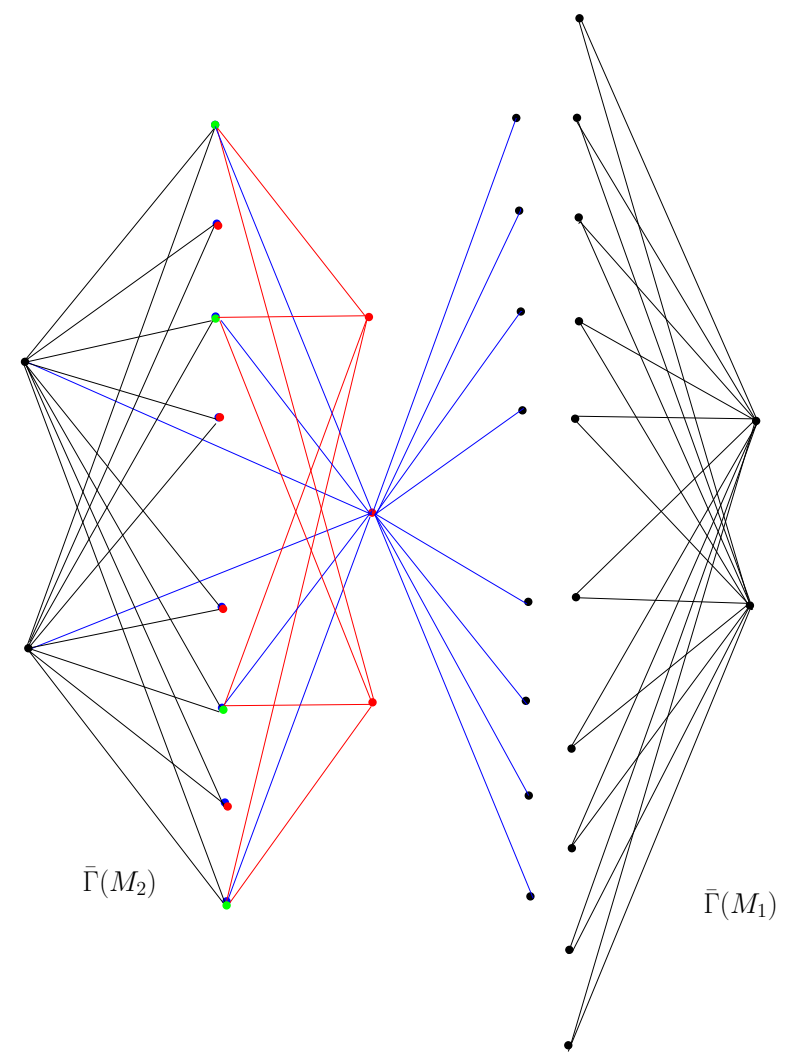

FigURE 4

\section{References}

[1] D. D. Anderson, Multiplication ideals, multiplication rings and the ring $R(X)$, Canad. J. Math. 28 (1976), no. 4, 260-768.

[2] D. D. Anderson and M. Naseer, Beck's coloring of a commutative rings, J. Algebra 159 (1993), no. 2, 500-514.

[3] D. F. Anderson, M. C. Axtell, and J. A. Stickles, Zero-divisor graphs in commutative rings, Commutative algebra, Noetherian and non-Noetherian perspectives, 23-45, Springer, New York, 2011.

[4] D. F. Anderson and A. Badawi, The total graph of a commutative ring, J. Algebra 320 (2008), no. 7, 2706-2719.

[5] - On the zero-divisor graph of a ring, Comm. Algebra 36 (2008), no. 8, 30733092.

[6] D. F. Anderson, A. Frazier, A. Lauve, and P. S. Livingston, The zero-divisor graph of a commutative ring. II, Ideal theoretic methods in commutative algebra (Columbia, MO, 1999), 61-72, Lecture Notes in Pure and Appl. Math., 220, Dekker, New York, 2001.

[7] D. F. Anderson, R. Levy, and J. Shapiro, Zero-divisor graphs, von Neumann regular rings, and Boolean algebras, J. Pure Appl. Algebra 180 (2003), no. 3, 221-241. 
[8] D. F. Anderson and P. S. Livingston, The zero-divisor graph of a commutative ring, J. Algebra 217 (1999), no. 2, 434-447.

[9] A. Badawi and D. F. Anderson, Divisibility conditions in commutative rings with zerodivisors, Comm. Algebra 38 (2002), no. 8, 4031-4047.

[10] A. Barnard, Multiplication modules, J. Algebra 71 (1981), no. 1, 174-178.

[11] I. Beck, Coloring of commutative rings, J. Algebra 116 (1988), no. 1, 208-226.

[12] G. A. Cannon, K. Neuerburg, and S. P. Redmond, Zero-divisor graphs of nearrings and semigroups, Nearrings and nearfields, 189-200, Springer, Dordrecht, 2005.

[13] F. R. DeMeyer, T. McKenzie, and K. Schneider, The zero-divisor graph of a commutative semigroup, Semigroup Forum 65 (2002), no. 2, 206-214.

[14] R. Diestel, Graph Theory, Springer-Verlag, New York, 1997.

[15] Z. A. El-Bast and P. F. Smith, Multiplication modules, Comm. Algebra 16 (1988), no. $4,755-779$.

[16] SH. Ghalandarzadeh and P. Malakooti Rad, Torsion graph over multiplication modules, Extracta Math. 24 (2009), no. 3, 281-299.

[17] J. Han, The zero-divisor graph under group actions in a noncommutative ring, J. Korean Math. Soc. 45 (2008), no. 6, 1647-1659.

[18] F. Kash, Modules and Rings, London: Academic Press, 1982.

[19] P. Malakooti Rad, Sh. Ghalandarzadeh, and S. Shirinkam, On the torsion graph and von Neumann regular rings, Filomat 26, 47-53. To appear.

[20] H. Matsumara, Commutative Ring Theory, Cambridge, UK: Cambridge University Press, 1986

[21] S. P. Redmond, The zero-divisor graph of a non-commutative ring, Internat. J. Commutative Rings 1 (2002), no. 4, 203-211.

[22] P. Ribenboim, Algebraic Numbers, Wiley, 1972.

Shaban Ghalandarzadeh

Department of Mathematics

FACUlty of SCIENCE

K. N. Toosi University of Technology

TEHRAN 16315-1618, IrAN

E-mail address: ghalandarzadeh@kntu.ac.ir

Parastoo Malakooti Rad

Faculty of Electronic and Computer and IT

ISLAMIC AZAD UNIVERSITY

QAZvin Branch, QAZvin 1416-3418, Iran

E-mail address: pmalakoti@gmail.com

SARA ShIRINKam

Department of Mathematics

FACUlTy OF SCIENCE

K. N. Toosi University of Technology

TEHRAN 16315-1618, Iran

E-mail address: sshirinkam@dena.kntu.ac.ir 